\title{
Qualität muss sich lohnen
}

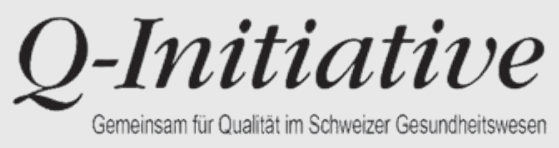

\author{
Die vier Organisationen (EQUAM Stiftung, Schweizerischer Dachverband der Ärzte- \\ netze medswiss.net, die Argomed Ärzte AG und der Verband deutschschweizeri- \\ scher Ärztegesellschaften VEDAG), die sich im Februar 2013 zur «Q-Initiative» zu- \\ sammengeschlossen haben, zeigen auf, in welchen Bereichen sie aktiv geworden \\ sind und nehmen Stellung zum geplanten Zentrum für Qualität.
}

\section{Ignazio Cassis ${ }^{a}$ Wolfgang Czerwenka ${ }^{b}$, Christoph Ramstein ${ }^{\text {, }}$ Rainer Hurnid}

a Dr. med., Nationalrat, Präsident curafutura, Präsident EQUAM Stiftung (Externe Qualitätssicherung in der ambulanten Medizin) Q-Module

b Dr. med., medizinischer Geschäftsleiter, Argomed Ärzte AG - Trägerschaft Q-Label MehrFachArzt; Schweiz. Dienstleistungsunternehmen für medizinische Versorgungsmodelle und Ärztenetze

c Dr. med., Co-Präsident VEDAG und Projektleiter QBM - QBM (Qualitäts-BasisModul), Verband Deutschschweizerischer Ärztegesellschaften

d Dr. med., Vorstand medswiss. net, Ressort Qualität - QMN (qualimed-net), Schweizer Dachverband der Ärztenetze

* Die Q-Initiative und die dahinterstehenden Organisationen wurden im letzten Jahr in der SÄZ vorgestellt: Cassis I, Czerwenka W, Ramstein C, von Weymarn A. Qualität in der ambulanten Medizin: Zusammen ist man stärker. Schweiz Ärztezeitung. 2013;94(10): 371-2.

\section{Korrespondenz:}

Q-Initiative

c/o EQUAM Stiftung

z.H. Sigrid Hess-Scheurer

Effingerstrasse 25

CH-3008 Bern

Tel. 0612710111

sigrid.hess[at]equam.ch

\section{Qualität muss sich lohnen}

Qualität ist in aller Munde, doch unter diesem Begriff versteht nicht jeder das Gleiche. Das Wort hat eine Bedeutung für Patienten, eine andere für Leistungserbringer - mit grossen Unterschieden, ob es sich um Ärzte, Apotheker, Spitäler oder andere Vertreter des Gesundheitswesens handelt; und nochmals anders interpretieren Versicherer und Politiker den Begriff Qualität. Wegen dieser Komplexität wurde in unserem liberalen Gesundheitswesen die Sicherstellung der Qualität an die Vertragspartner delegiert. Gemäss KVG sollen diese, gemeinsam und auf vertraglicher Basis (ohne dass sich der Staat zu stark einmischt), die Qualität sichern und sie kontinuierlich verbessern.

Doch nach 18 Jahren KVG ist die Bilanz bescheiden, vor allem in der ambulanten Versorgung. Durch die höhere intrinsische Motivation haben die Ärztenetze der Qualität mehr Aufmerksamkeit geschenkt als dies die Einzelpraxen getan haben, doch auch hier gibt es erste Ermüdungserscheinungen. Denn sobald die Qualitätskriterien in die Arbeitsprozesse integriert sind, ist das Ziel erreicht. Warum weiterhin Gelder in die Qualität investieren, wenn dadurch kein Wettbewerbsvorteil entsteht? Im aktuellen System fehlen geeignete Anreize, um Beweise für die erbrachte Qualität zu liefern. Qualität muss sich eben lohnen!

Die hier vorgestellte Q-Initiative ist am 7. Februar 2013 in Luzern entstanden: Vier anerkannte Organisationen - Argomed Ärzte AG, EQUAM Stiftung, medswiss.net und VEDAG - haben entschieden, ihre Kräfte zu bündeln, um gemeinsam die Qualitätssicherung in der ambulanten Medizin zu fördern. Wird dadurch der Sprung in eine neue Q-Ära gelingen? So einfach ist es nicht, solange Qualität sich finanziell nicht wirklich lohnt. Wenn nur Kosten entstehen, werden sogar Angefressene müde.

Doch ist Bundesbern nicht bereit, noch lange zu warten. 2007 hat das Parlament entschieden, der Bundesrat müsse die Führung übernehmen und von seiner subsidiären Rolle Gebrauch machen (ähnlich wie kürzlich im TARMED-Bereich). Das BAG hat seit 2009 fleissig gearbeitet: Ein neues Bundesgesetz über das Zentrum für Qualität in der OKP war kürzlich in der Vernehmlassung. Sollen wir uns auf den Staat verlassen oder selber aktiv werden? Unsere vier Organisationen haben diesen zweiten Weg gewählt und versuchen, selber die Hausaufgaben zu erledigen. Auch die FMH ist Ende 2012 mit der SAQM (Schwei-

\section{Die Partner der Q-Initiative}

\section{EQUAM}

EQUAM fördert die externe, unabhängige Qualitätssicherung in der ambulanten ärztlichen Versorgung. Als akkreditierte Zertifizierungsstelle, welche sich der Unparteilichkeit verpflichtet hat, verleiht EQUAM ein Qualitätszertifikat für Arztpraxen, das EQUAMLabel für medizinische Qualität und Patientensicherheit.

www.equam.ch

\section{Argomed - MehrFachArzt}

MehrFachArzt zeichnet besonders kompetente Hausärztinnen und Hausärzte aus. Das Gütezeichen garantiert die breite ärztliche Aus- und Weiterbildung sowie die stetige Fortbildung, aber auch kompetentes Praxispersonal und eine gute Praxiseinrichtung.

www.mehrfacharzt.ch

\section{quali-med-net}

quali-med-net erlaubt integrierten Ärztenetzwerken mit einem netzspezifischen Indikatorenset, eigene gesetzte Qualitätsziele im Längsverlauf festzulegen und zu überprüfen, und sich gleichzeitig im Quervergleich mit andern Netzwerken kompetitiv zu vergleichen.

www.medswissnet.ch

\section{VEDAC - QBM}

QBM, das Qualitäts-Basis-Modul macht die ärztliche Qualität in der Praxis sichtbar. Mit QBM können Ärztinnen und Ärzte sich selber und das Praxisteam testen, Abläufe in der Praxis bewerten und Patienten befragen. Sie erfassen ihre Leistungen systematisch und erhalten Auswertungen mit individuellen Empfehlungen im Längs- und Quervergleich. So sichert und fördert QBM die ärztliche Qualität im Sinne der Qualitätsentwicklung.

www.vedag-qbm.ch 
zerische Akademie für Qualität in der Medizin) in diesem Feld aktiver geworden. Doch eine Schwalbe macht noch keinen Sommer!

Wir brauchen die aufgeklärte Unterstützung aller ambulant tätigen Ärzte und der Krankenversicherer, um gemeinsam die Qualitätssicherung in den Praxisalltag zum Durchbruch zu bringen.

\section{Bilanz Q-Initiative - 18 Monate nach der Gründung}

Die Idee zur Q-Initiative entstand 2012, deren Gründung erfolgte am 7. Februar 2013. Vier anerkannte Organisationen - Argomed Ärzte AG, EQUAM Stiftung, medswiss.net und VEDAG - haben entschieden, ihre Kräfte zu bündeln, um gemeinsam die Qualitätssicherung in der ambulanten Medizin zu fördern. Hierzu suchen wir geeigneten Partnerschaften mit Drittorganisationen. Die erste fruchtbare Partnerschaft wurde mit der Ärztekasse Genossenschaft geschlossen. Sie bietet ihren Kunden finanzielle Vorzugskonditionen an, wenn sie bei einem unserer vier Organisationen mitmachen und ab 2013 ein entsprechendes Qualitätslabel vorweisen können. Bis jetzt haben allerdings nur wenige teilnehmende Ärztinnen und Ärzte davon Gebrauch gemacht. Sind Sie interessiert? Dann melden Sie sich bei marketing[at]aerztekasse.ch.

Andere Anreize mit neuen Partnern sind zurzeit in Evaluation. Sobald neue Verträge unterschrieben sind, werden wir dies publik machen und auf den Webseiten der vier Organisationen entsprechend aufschalten.

Zudem pflegen wir einen regelmässigen Informationsaustausch mit halbjährlichen gemeinsamen Treffen: Wir versuchen somit, unsere verschiedenen Kulturen besser zu verstehen und miteinander in Einklang zu bringen. Dabei werden Vertrauen geschaffen, in gemeinsamen Schritten Aktivitäten geplant und Entscheide getroffen, wie z.B. die gemeinsame Stellungnahme zum neuen Bundesgesetz über ein Qualitätszentrum. Wir alle sind in einem wettbewerblichen Kontext eingebettet, der uns zwingt, innovativ zu werden und Schritt für Schritt das Qualitätsthema in der Ärzteschaft zu fördern, damit die gelebte Qualitätsentwicklung zu einer breiten Realität in der ambulanten Medizin der Schweiz wird.

\section{Zwischen Kooperation, Konkurrenz und Koordination}

\section{Stellungnahme der Q-Initiative zum geplanten Zentrum für Qualität}

Wie können wir eine hochwertige und wirtschaftliche Gesundheitsversorgung sicherstellen? Geht es nach dem Bund, so soll ein «Zentrum für Qualität» wesentliche Impulse dazu geben. Ein entsprechender Gesetzesentwurf durchläuft momentan in Begleitung eines erläuternden Berichts eine breite Vernehmlassung.
Beim Lesen wird einerseits klar, wie wichtig Qualitätsanstrengungen sind: Beispielhaft erwähnt der Bund, dass vermeidbare Zwischenfälle in der Versorgung rund 350000 Spitaltage pro Jahr verursachen. Der Handlungsbedarf ist gegeben. Doch andererseits entsteht der Eindruck, dass der Bund sich mit seinem Vorschlag nicht recht zu entscheiden weiss zwischen Konkurrenz, Kooperation und Koordination.

Die Möglichkeit zur Konkurrenz zu bestehenden Qualitätsinitiativen tut sich in Bezug auf die inhaltliche Arbeit auf: "Das Zentrum selbst hat die Aufgabe, die vom Bundesrat verabschiedeten Programme zu planen, durchzuführen und zu evaluieren», heisst es im Bericht (S. 35). Aber auch: «Die neu durch den Bund aufgenommenen Aktivitäten ersetzen weder die Qualitätssicherung noch die laufenden Qualitätsbestrebungen der verschiedenen Partner im Gesundheitswesen» (S. 3). Die Widersprüchlichkeit dieser beiden Aussagen wird vor allem deutlich, wenn man sie aus der Perspektive bestehender Initiativen für Qualität und Patientensicherheit liest. Sie werden nämlich im Bericht kaum erwähnt und wohl nicht wirklich als Partner wahrgenommen. Doch wenn das Zentrum selbst inhaltliche Arbeit leistet, so kann eine Konkurrenzsituation nur durch eine konsequente Einbindung bestehender Initiativen vermieden werden. Kooperation müsste über Mitarbeit der Initiativen am Zentrum oder über Aufträge hergestellt und gepflegt werden.

Ein anderer Weg wäre eine stärker koordinative Positionierung des Zentrums, wie sie Alain Berset gegenüber der Presse erwähnt (Tagesanzeiger vom 15.5.2014). Doch auch diese Stossrichtung scheint beim Bund nicht ganz ausgereift. Eine wirkungsvolle inhaltliche und strategische Koordination der Qualitätsinitiativen nämlich benötigt als Grundlage eine Verbindlichkeit von Qualitätskontrollen, wie sie schon im Krankenversicherungsgesetz gefordert, aber kaum umgesetzt wird. Doch das Zentrum soll keinerlei hoheitliche oder regulative Funktionen wahrnehmen. Damit kann die Institution zwar zwischen bestehenden Initiativen, nicht jedoch zu den Organisationen des Gesundheitswesens hin, effizient koordinieren.

Gewissermassen platziert der Bund Subsidiarität am falschen Ort. Das, was professionelle private und halbprivate Institutionen teilweise heute schon leisten - nämlich die inhaltliche Qualitätsarbeit -, nimmt der Bund an sich, ohne bestehende Initiativen ernsthaft mit einzubeziehen. Das, was die bestehenden Q-Initiativen jedoch sicher nicht können und die Tarifpartner jahrelang verschlafen haben nämlich Verbindlichkeiten herstellen -, will der Bund nicht in Angriff nehmen.

Eine Klärung ist nötig. Aus Sicht der Q-Initiativen: Konkurrenz? Wohl kaum eine wirksame Strategie. Kooperation? Ja, aber gerne auf Augenhöhe. Koordination? Unbedingt, auf verbindlicher Basis. 


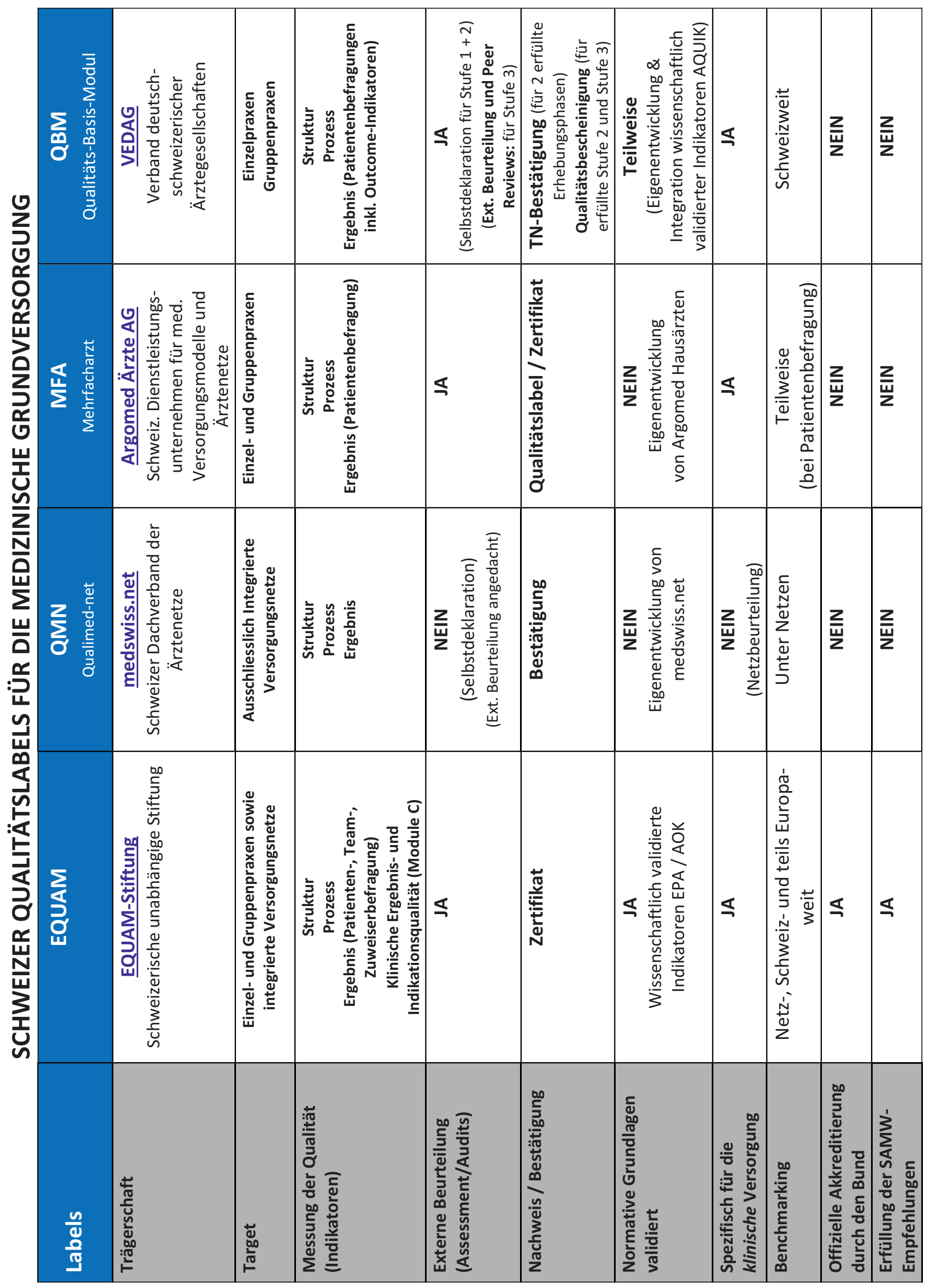

